\title{
Health literacy and age-related health-care utilisation: a multi-dimensional approach
}

\author{
Joachim Gerich $^{1 *}$ (D), Robert Moosbrugger ${ }^{1}$ and Christoph Heigl ${ }^{2}$ \\ ${ }^{1}$ Department of Sociology, Johannes Kepler University Linz, Linz, Austria and ${ }^{2}$ Upper Austrian Sickness \\ Fund, Linz, Austria \\ ${ }^{*}$ Corresponding author. Email: joachim.gerich@jku.at
}

(Accepted 21 October 2020; first published online 23 November 2020)

\begin{abstract}
Inefficient health service utilisation puts pressure on health systems and may cause such negative individual consequences as over-medicalisation or exacerbation of health problems. While previous research has considered the key relevance of health literacy (HL) for efficient use of health services, the results of that research have been somewhat inconclusive. Possible reasons for diverging results of prior research may be grounded in different measurement concepts of HL and the disregarding of age-specific effects. This paper analyses the association between individuals' HL typology based on a two-dimensional concept and indicators of health service utilisation measured by registered data covering the number of doctor visits and medication costs. Our results confirm a significant interaction effect between age and HL typology. The age-related increase in health service utilisation is strongest for individuals with the combination of high subjective HL but low health-related knowledge, while the smallest increase is for individuals with the constellation of high subjective HL combined with high health-related knowledge. Individuals with specific constellations of HL (that is, individuals with high subjective HL but low healthrelated knowledge) are associated with reduced service utilisation in younger ages but higher service utilisation in later stages of life, compared to other groups. These results are likely to be attributed to a higher external health-related locus of control and more traditional paternalistic role expectations in such groups.
\end{abstract}

Keywords: health literacy; health-related knowledge; medication; health service utilisation; health; doctor visits

\section{Introduction}

Inefficient use of health-care services increases financial pressure of care systems, reduces the capacity of health-care services and may promote unnecessary medication of patients. Especially in light of demographic trends (population ageing) and epidemiologic transitions (increasing prevalence of chronic diseases), identifying and addressing factors associated with efficient use of health-care services is a 
striking issue. In recent decades, scholars have described the patient empowerment and health literacy (HL) of individuals as an important prerequisite for efficient health service utilisation (Berkman et al., 2011; Palumbo, 2017). The aim of the present study is to analyse the potentially moderating role that HL plays in the common age-related increase in health service utilisation, as measured by registered doctor visits and medication costs in a sample of a general population aged between 18 and 80 years.

\section{$H L$ and health service utilisation}

Based on an extensive literature review, Sørensen et al. defined HL broadly as

people's knowledge, motivation and competences to access, understand, appraise, and apply health information in order to make judgments and take decisions in everyday life concerning healthcare, disease prevention and health promotion to maintain or improve quality of life during the life course. (Sørensen et al., 2012: 3)

Sufficient HL is viewed as a prerequisite to health in that it enables individuals to actively engage in self-care, navigate through complex health service systems, acquire and process health-related information and make informed health-related decisions (Paasche-Orlow and Wolf, 2007; Palumbo, 2017). HL is considered an important factor regarding individual health behaviours as well as health outcomes (Goto et al., 2019), while low HL has been found to be related to overutilisation and under-utilisation of health services (Haun et al., 2015; Goto et al., 2019). Apart from positive effects on health, it is assumed that sufficient $\mathrm{HL}$ is associated with a more efficient utilisation of health-care services, reduced medicalisation and hence reduced health-related expenses. Previous studies have confirmed the higher medical costs of individuals and more inefficient health service utilisation (such as higher numbers of doctor visits and hospitalisation) among those with limited HL (Baker et al., 1998; Howard et al., 2005; Cho et al., 2008; Ishikawa and Yano, 2008; Eichler et al., 2009; Berkman et al., 2010, 2011; Haun et al., 2015; Vandenbosch et al., 2016; MacLeod et al., 2017; Palumbo, 2017). Contrary to higher medication and curative health service utilisation, less utilisation of preventive services like screenings and vaccination has been found to be associated with low HL (Ishikawa and Yano, 2008; Berkman et al., 2011; Vandenbosch et al., 2016). However, previous research has also produced inconsistent results regarding the association between HL and health-care costs (Eichler et al., 2009; Berkman et al., 2011; MacLeod et al., 2017). Vandenbosch et al. (2016), who analysed the association between HL and health service utilisation based on registry data, found higher costs and odds for some indicators of service utilisation associated with low HL (psychiatric consultation, ambulance transports, psychiatric hospital stays, higher number of hospital days, higher numbers of doctor visits), but lower medication costs for those with insufficient HL. Similarly, Berens et al. (2018) reported heterogeneous findings regarding the association between HL and health service utilisation, and suggested that part of this variation is due to different measures of HL and different sets of considered control variables. 
There are strong variations regarding the concepts and measurement strategies of HL in previous research. Despite the broad theoretical definition of HL, most of the research has focused on measures of functional HL, which predominately focuses on basic cognitive skills of comprehension and numeracy (Berkman et al., 2011; Sørensen et al., 2013; Palumbo, 2017). Therefore, Palumbo (2017: 210) not only questioned the comparability of previous findings regarding the association between HL and health service utilisation, but also argued that results may be biased 'due to the disregard of the role played by both interactive and critical health literacy skills'. According to Nutbeam (2008), interactive HL covers the social skills and motivation necessary for successful interactions with health-care providers and critical HL is necessary for the appraisal of information and recommendations provided by health-care providers or via media.

\section{Measures of $H L$}

Covering a broader spectrum of HL was the underlying idea when the HLS-EU-Q inventory was developed (HLS-EU Consortium, 2012). The inventory intends to cover a broader public health perspective of $\mathrm{HL}$ in the sense of empowerment, also including components of critical and interactive HL (HLS-EU Consortium, 2012; Sørensen et al., 2012). Therefore, it asks for respondents' difficulty estimations of various health-related tasks, covering the domains of health care, disease prevention and health promotion. Contrary to frequently used measures of functional HL, the HLS-EU-Q inventory focuses on subjective judgements regarding respondents' ability to accomplish situational health-related demands (Sørensen et al., 2015). Using a Likert-type response scale ('On a scale from very easy to very difficult, how easy would you say it is to...'), the inventory covers subjective difficulty estimations regarding tasks such as understanding what a doctor says, finding and understanding information for health prevention, or judging the reliability of health-related information in the media. Responses to the 47 items of the long version (16 items of the short version) are used to calculate an overall HL score.

Although it is acknowledged that the HLS-EU-Q covers a broader concept of literacy beyond simple cognitive abilities, some scholars have raised concerns regarding the validity of the inventory (Bitzer, 2017; Steckelberg et al., 2017; Samerski, 2019). The main critique refers to possible misclassifications associated with subjective HL scores. It is argued that, even for medical experts, some health-related tasks included in the HLS-EU-Q, such as correct understanding of medical leaflets or judgements regarding the benefits of early detection screenings are difficult to manage. Empowered individuals with a high HL may rate these tasks as difficult, because they are aware that they require intensive weighting of pros and cons, although this will result in low HL scores. On the contrary, respondents who judge these tasks as easy to accomplish (resulting in high HL scores) may do so due to an overestimation of their own competences, habitualised compliance or low reflectivity, combined with an underestimation of the difficulty of the task at hand. Using the HLS-EU-Q to analyse differences in health-care service use in Belgium, Vandenbosch et al. (2016) found associations between low HLS-EU-Q scores and higher numbers of admissions to one-day clinics, home and psychiatrist consultations, and ambulance transports. No associations were found concerning 
specialist consultation, consultations of general practitioners or emergency consultations. The findings concerning medication use are inconsistent. For Germany, Berens et al. (2018) found differences in the utilisation of curative health services, where low HL measured by the HLS-EU-Q was associated with more frequent usage. However, when controlling for socio-demographic and health-related factors, only small significant effects regarding visits to doctors and other health professionals were confirmed.

In a mixed-method study, Gerich and Moosbrugger analysed the validity of the HLS-EU-Q and concluded that some individuals scoring high on this inventory may in fact be highly empowered as they have the 'competences to access, understand, appraise and apply health information in order to make informed health-related decisions' (Gerich and Moosbrugger, 2018: 257). In the same study, however, some respondents scored high on the HLS-EU-Q measure even though their empowerment and health-related competences are low. In these cases, the high score for subjective HL contrarily mirrors high trust in physicians and high external locus of control, which results in the optimistic expectation that dealing with health problems is 'easy' as long as one trusts in medical experts. To address this heterogeneity of HL scores, Gerich and Moosbrugger (2018) suggested considering a HL typology by combining subjective HL scores with a measure of health-related knowledge. They found that individuals with high health-related knowledge and high scores on subjective HL tend to be active, motivated and critical patients who prefer interactive doctor-patient interactions over traditional paternalistic role situations. By contrast, those individuals with high scores on subjective HL but low health-related knowledge tend to prefer more traditional passive roles in doctor-patient interactions, show higher external locus of control, and high trust in the competence of the health-care system and its medical experts. Moreover, individuals with this HL constellation reported less active health-related coping styles, less willingness for self-medication but higher degrees of compliance with physicians' recommendations. Although high unconditional trust and passive roles may be functional for the maintenance of health when personal health-related resources are low, the high subjective HL score of these individuals does not mirror the idea of empowered patients that actively make their own informed judgements and health-related decisions.

Hence, with respect to health service utilisation, different outcomes depending on the constellation of health-related knowledge and subjective HL could be expected. Efficient health service use and reduced costs associated with high HL, as described above, may be expected for empowered and reflective individuals corresponding with the constellation of high subjective HL, combined with sufficient health-related knowledge. On the contrary, for individuals characterised by a constellation of high subjective HL paired with low health-related knowledge different outcomes are expected as a result of their more paternalistic role expectations, high external locus of control and higher trust in the expertise of medical experts. More precisely, it could be expected that individuals with this type of HL constellation will consult physicians in cases of health complaints more frequently and that due to their preferred passive role and their higher preference for medication the outcomes of doctor-patient interactions will be less efficient. Hence, it is expected that health service utilisation and medication costs are higher in the 
constellation of high subjective HL paired with low health-related knowledge. Moreover, as people with this HL constellation have a higher focus on passive curative actions and less preference for active health promotion, a higher risk of exacerbation of health problems in later stages of life could be expected.

\section{The role of age}

The present study has a particular focus on age, which is predominantly grounded on three factors.

First, there is reason to assume that age is a potential confounder for associations between HL and service utilisation or health-care costs. There is ample evidence for increasing disease burden in higher ages (mostly defined as 65 years or older), which means that health service utilisation will increase with age (Nie et al., 2010). Moreover, associations between age and HL have to be assumed (Vogt et al., 2019), even though previous research is inconclusive in this respect; whereas some studies have found that HL increases with age, others have reported the opposite (Tiller et al., 2015). Tiller et al. (2015) pointed out that HL may be expected to decrease with higher age due to decreasing cognitive capacity, which could mainly be argued with respect to functional HL. However, increasing HL with higher age could be assumed given that older people are more concerned with health issues due their higher disease burden. Hence, higher health-related knowledge will be acquired as a consequence.

Kobayashi et al. (2016) argue that there is evidence for age-related declines of fluid cognitive abilities (e.g. working memory, reasoning, problem solving), but that crystallised abilities (e.g. generalised knowledge, vocabularies) are likewise stable across the age-span. On the basis of their meta-analysis, they found some evidence for their hypothesis that the age-related decline in HL depends on the type of HL assessments used. Whereas stronger age-related declines in HL are confirmed for functional HL tests that rely strongly on abilities regarding numeracy or problem solving (fluid cognitive abilities), functional HL remains more stable for assessments that rely on medical vocabulary (crystallised abilities).

A second reason for the special consideration of age is that elderly people (65 years or older) account for the largest proportion of health-care service utilisation (Nie et al., 2010). Together with demographic changes and epidemiological transitions to chronic diseases in Western countries, health-care utilisation of elderly people is a major concern (Parker et al., 2003; MacLeod et al., 2017; Palumbo, 2017). As Nie et al. (2010) pointed out, age is not only associated with increased health-care utilisation, but also with an increase in the variance of health-care utilisation. HL may be one of the relevant factors to explain this higher variance in health-care utilisation among elderly people. The higher prevalence of chronic diseases in later life is associated with higher requirements regarding navigation through the health system, dealing with health-related decisions and the management of more complex therapies (Vogt et al., 2019). Therefore, as HL is a prerequisite for successful coping with these demands, it is expected that the significance of sufficient HL for efficient health service utilisation and self-management increases with age (Haun et al., 2015).

Third, although low HL was found to be associated with worse health outcomes, this association is expected to increase with higher age due to cumulative effects. 
More precisely, low HL was found to be associated with reduced motivation for and practice of health-promoting behaviour, less symptom attention (Taib et al., 2011; Reisi et al., 2014; Yang et al., 2017; Fleary et al., 2018), as well as poorer medication adherence and less health service utilisation regarding disease prevention or health promotion (Berkman et al., 2010; Vandenbosch et al., 2016; Palumbo, 2017; Berens et al., 2018). Hence, although low HL can be expected to be associated with a higher number of risk factors for worse health outcomes, manifest consequences are likely to be observed in later stages of the lifecourse due to the time-lagged effects of these risk factors. Moreover, as insufficient HL is expected to negatively affect health states in later years, and low HL is associated with less-efficient use of health services to cope with these increased health-related demands, a 'spiralling' effect of insufficient $\mathrm{HL}$ in later years with respect to health service utilisation and medication costs could be expected (Palumbo, 2017).

Taken together, heterogeneity regarding the association between HL and health service utilisation depending on individuals' age could be expected. Health service utilisation of younger individuals with insufficient HL may be low due to lower motivation for health promotion and less attention to self-care. However, health service demands may increase disproportionately in older age due to cumulative health effects and insufficient health service utilisation. Consequently, an interaction effect between age and HL with respect to health service utilisation is expected.

\section{Research questions}

The aim of our research is to analyse the association between HL types and health service utilisation. Moreover, the research is based on the general presumption that health service utilisation increases with higher age of individuals due to higher disease burden and increasing prevalence of chronic conditions in later stages of life, leading to the following hypothesis:

- Hypothesis 1: Health service utilisation (number of doctor visits and costs for medication) increases with higher age.

However, as previous research has found higher variance of health service utilisation of elderly people compared to younger people, we assume that part of this variance is explained by different constellations of HL. It is expected that individuals with high subjective HL and high health-related knowledge have higher skills necessary for efficient health service utilisation, as well as higher competence for self-help regarding health complaints. On the other hand, individuals with high subjective HL paired with low health-related knowledge are expected to have low competences for self-help and be more dependent on medical experts combined with high trust in the medical system. Consequently, the common increase in health service utilisation with higher age is expected to differ with respect to these two types of HL; this leads to Hypothesis 2, which includes an interaction effect between age and HL type:

- Hypothesis 2: The extent of age-related increase in health service utilisation depends on the HL type, such that-compared to individuals with high 
subjective HL paired with high health-related knowledge - a higher increase is observed for individuals with the constellation of high subjective HL and low health-related knowledge.

\section{Method}

\section{Participants and procedure}

The data for this study were gathered between January and February 2017 through a mailed questionnaire that was sent to a random sample of 3,500 individuals drawn from the register of the Upper Austrian sickness fund, which provides the mandatory health insurance for about 75 per cent of the 1.5 million inhabitants of the province of Upper Austria. Five hundred and ninety respondents aged between 18 and 80 years with valid questionnaire information who gave their consent for their responses to be matched with registered data on health service use were used for the analyses. For each respondent, information on health service utilisation (number of doctor visits and costs for medication) was taken from the registered records. Compared to the target population, females (56.8\% versus $51.9 \%$ in the target population) and older persons (58.8\% are older than 50 , compared to $40.3 \%$ overall) are overrepresented in the sample population. To control whether our results are affected by this sample bias, all analyses were replicated with poststratifications according to the combined distribution of age and gender. Some minor differences between weighted and unweighted analyses were observed. Differences regarding the significance of the estimated parameters are indicated in the text.

\section{Measures}

The HL typology was computed as described in Gerich and Moosbrugger (2018). For this purpose, subjective HL was measured with the 16-item short version of the HLS-EU-Q (e.g. 'On a scale from very easy to very difficult, how easy would you say it is to: find information on treatments of illnesses that concern you?') (Sørensen et al., 2013, 2015). The mean score across the 16 items ranging from 1 (low HL) to 4 (high HL) was computed as the measure for subjective HL (mean $=2.91$, standard deviation $(\mathrm{SD})=0.44)$. Items for the health-related knowledge scale were taken from Gerich and Moosbrugger (2018). Due to limited space in the questionnaire, only five out of 13 items were used. These items consist of statements for which respondents were asked to indicate whether they believe they are 'true', 'false' or whether they 'don't know' if they are true or false. The items were (a) 'the term Hepatitis labels an inflammation of the heart muscle', (b) 'a medical specialist for diseases of the heart is referred to as a cardiologist', (c) 'only children may suffer from whooping cough', (d) 'antibiotics reduce disease duration of severe colds', and (e) 'more people in Austria die from coronary heart disease than from malignant tumours (cancer) annually'. The number of correct answers was used to compute a measure for health-related knowledge. The average number of correct answers given by the participants was $3.4(\mathrm{SD}=1.26)$. To construct the HL typology, both measures were dichotomised at the median of the distribution (which was 4 for knowledge and 3 for the subjective HL) to achieve a sufficient sample size of 
the resulting sub-groups. Subsequently, a HL typology was constructed from the two measures (see Table 1):

- Respondents with high subjective HL and high health-related knowledge are denoted as HL Type 4.

- Those with low subjective HL and low health-related knowledge are denoted as HL Type 1.

- Respondents with high subjective HL but low health-related knowledge are denoted as HL Type 3.

- Those with low subjective HL but high health-related knowledge are denoted as HL Type 2.

Because subjective HL and health-related knowledge are only weakly correlated $(r=0.07 ; p>0.05)$, a well-balanced distribution of the four HL types is observed, which is in accordance with previous results (Gerich and Moosbrugger, 2018).

Median splits have been criticised by some scholars to produce biased results due to increasing Type I and Type II errors in statistical analyses. However, as shown by Iacobucci et al. $(2015 a, 2015 b)$, median splits do not affect the robustness of analyses, when both variables are uncorrelated and sample size is not too small. As both preconditions hold for the present study, the use of a median split in constructing our HL typology is acceptable.

Questionnaire responses were matched with data about health service utilisation taken from patient records of the Upper Austrian sickness fund. The data cover respondents' health service utilisation over the past year.

The number of doctor visits is the registered number of respondents' consultations with general practitioners within the past year. Medication costs are the total costs of prescribed medications for each individual within the past year gathered from registered data. These medications include filled and dispensed prescriptions from general and specialist physicians as well as those from hospitals, but exclude drugs directly given to patients in emergency departments.

To avoid bias due to extreme values, 2 per cent of respondents with the highest registered medication costs and number of doctor visits based on the cumulative distribution were excluded from the analyses.

The focus of our research is on aspects of health service utilisation rather than specific health outcomes associated with HL. Nevertheless, as it is expected that health service utilisation is predominately dependent on individuals' health, which is again expected to be related to HL, subjective estimation of health status is considered as a control variable with which to analyse differences in health-care utilisation dependent on HL types. Subjective self-rated health was measured with a single-item question where respondents rate their health condition on a scale ranging from 0 (worst imaginable health) to 10 (best imaginable health) (Nübling et al., 2005).

Similarly, HL, health status and health service utilisation are known to be dependent on individuals' socio-economic status and gender (Nie et al., 2010; Bähler et al., 2015; Haun et al., 2015; Palumbo, 2017; Berens et al., 2018; Vogt et al., 2019). Therefore, gender and self-rated socio-economic status are considered as additional control variables. 
Table 1. Health literacy (HL) typology

\begin{tabular}{ccc}
\hline & Low health-related knowledge & High health-related knowledge \\
\hline Low subjective HL: & HL Type 1 & HL Type 2 \\
\hline$\%$ & 26.4 & 28.0 \\
\hline N & 156 & 165 \\
High subjective HL: & HL Type 3 & HL Type 4 \\
\hline$\%$ & 19.5 & 26.1 \\
\hline N & 115 & 154 \\
\hline
\end{tabular}

Notes: Sizes of sub-groups defined by the combination of the level of subjective $\mathrm{HL}$ and health-related knowledge. Low scores are defined as values below the median, high scores are defined as values greater or equal to the median.

Subjective socio-economic status was measured with the German version of the McArthur Scale (Adler et al., 2000; Hoebel et al., 2015), for which high consistence with objective measures of social status, as well as health-related construct validity, has been confirmed. The measure consists of a drawn 'ladder' with ten steps representing respondents' subjective positions in society. The highest step (10) corresponds to those people 'who have the most money, most education and best jobs'. The lowest step (1) represents the people with the 'least money, least education and worst jobs or no jobs'.

\section{Statistical analysis}

Analysis of variance and $\chi^{2}$-tests were used to analyse the differences between the four HL types. For the multivariate analyses, two-part hurdle models using generalised linear models were performed. Because the numbers of doctor visits represents count data resulting in heavily skewed distributions and a large proportion of respondents with values of zero (i.e. 'non-users'), the application of ordinary least square (OLS) regressions is not appropriate (Mihaylova et al., 2011). Instead, regression models appropriate for count data, such as Poisson or negative binomial models, can be considered. Due to over-dispersion - the variance of the number of doctor visits is clearly larger compared to the mean - a negative binomial model was used instead of the Poisson model. Moreover, due to the large proportion of respondents without doctor visits (19.2\%), a two-part (hurdle) model was calculated in which the users and non-users are analysed with separate models. Hence, for the first part of the hurdle model, a binary logistic regression model is used to analyse the association between the considered predictors and health service use (i.e. the number of doctor visits $>0$ versus 0 ). A negative binomial model with logarithmic link function for the number of doctor visits within the group of users (doctor visits $>0$ ) is performed with respect to part two of the model (Diehr et al., 1999; Mihaylova et al., 2011).

Similarly, OLS regressions are not appropriate for the analysis of medication costs because these distributions are also typically skewed, which means that heteroskedasticity, non-normal residuals and non-linear relationships could be expected (Barber and Thompson, 2004). In addition, similar to health-care utilisation 
data, a larger proportion of individuals with zero cost values must be considered (29.1\% in our sample). Therefore, a two-part model is also used for the analysis of medication costs. Again, a logistic regression model is used for the first part (i.e. cost $>0$ versus 0 ). A Gamma model with a log-link function is used for the costs in the second part of the model (for all costs > 0) (Diehr et al., 1999; Barber and Thompson, 2004; Mihaylova et al., 2011). Robust standard errors are computed for both generalised linear models in order to correct for heteroskedasticity due to moderate deviations from model assumptions (Barber and Thompson, 2004).

To test the assumed interaction effects between age and HL type, interaction terms are introduced to the regression models by multiplying $z$-standardised age with the non-redundant dummies.

\section{Results}

Table 2 provides descriptive variable information for the total sample and each of the four HL types.

As expected, self-rated socio-economic status and subjective health ratings differ significantly by HL type. Individuals classified as HL Type 4 report the highest social status as well as the best subjective health status. Also as expected, there is a significant variation in the number of doctor visits with respect to HL type with the highest number of doctor visits observed for HL Type 3. No overall significant differences with respect to HL type are observed for age, sex and medical costs.

The results of the bivariate and multivariate analyses with respect to doctor visits are shown in Table 3.

With respect to the zero-order correlations, the number of doctor visits increases with higher age, worse subjective health status and lower subjective socio-economic status. Moreover, compared to all other types, for individuals classified as HL Type 3 , a higher number of doctor visits can be observed. However, compared to all other types, a lower number of doctor visits is observed for individuals classified as HL Type 4 (not significant in analyses weighted by age and sex).

Turning to the multivariate analysis, the first part of the hurdle model shows only a significant effect for subjective socio-economic status. According to this result, a lower odds value for doctor visits over the past year is estimated for individuals with higher subjective socio-economic status adjusted for all other variables. In the model weighted by age and sex, an additional significant effect of subjective health is observed with lower odds for doctor visits for those with better health.

In the second part of the model it is confirmed that the number of doctor visits increases with higher age, worse subjective health and being classified as HL Type 3 compared to HL Type 4. Moreover, a positive significant interaction effect between age and HL Type 3 is confirmed, which is illustrated in the interaction plot in Figure 1.

The plot shows a significant stronger age-related increase in the number of doctor visits in individuals classified as HL Type 3 compared to those classified as HL Type 4. Moreover, due to the significant main (average) effect of HL Type 3, the number of doctor visits is higher for HL Type 3 independent of age, except for very young ages. 


\begin{tabular}{|c|c|c|c|c|c|c|c|c|c|c|c|}
\hline & \multicolumn{2}{|c|}{ Total sample } & \multicolumn{2}{|l|}{ HL Type 1} & \multicolumn{2}{|l|}{ HL Type 2} & \multicolumn{2}{|l|}{ HL Type 3} & \multicolumn{2}{|l|}{ HL Type 4} & \multirow[b]{2}{*}{$p^{1}$} \\
\hline & Mean (SD) or \% & N & Mean (SD) or \% & N & Mean (SD) or \% & N & Mean (SD) or \% & $N$ & Mean (SD) or $\%$ & $\mathrm{~N}$ & \\
\hline $\begin{array}{l}\text { Number of doctor } \\
\text { visits per year }\end{array}$ & $6.52(7.12)$ & 579 & $6.75(6.78)$ & 150 & $5.81(6.61)$ & 165 & $8.94(9.45)$ & 112 & $5.27(5.41)$ & 152 & $<0.001$ \\
\hline $\begin{array}{l}\text { Medication costs } \\
\text { (€ per year) }\end{array}$ & $176.86(353.64)$ & 581 & $225.60(401.62)$ & 155 & $165.86(380.35)$ & 163 & $190.12(310.11)$ & 114 & $128.07(292.12)$ & 149 & 0.107 \\
\hline Age & $52.02(15.93)$ & 590 & $52.04(16.50)$ & 156 & $51.90(15.05)$ & 165 & $52.61(17.65)$ & 115 & $51.70(15.02)$ & 154 & 0.973 \\
\hline Self-rated health ${ }^{2}$ & $7.07(2.06)$ & 590 & $6.60(2.07)$ & 156 & $7.01(2.09)$ & 165 & $6.98(2.06)$ & 115 & 7.69 (1.89) & 154 & $<0.001$ \\
\hline $\begin{array}{l}\text { Self-rated } \\
\text { socio-economic } \text { status }^{3}\end{array}$ & $5.69(1.60)$ & 590 & $5.31(1.49)$ & 156 & $5.60(1.52)$ & 165 & $5.37(1.68)$ & 115 & $6.39(1.53)$ & 154 & $<0.001$ \\
\hline Sex (\% female) & 56.8 & 590 & 55.1 & 156 & 57.6 & 165 & 49.6 & 115 & 48.4 & 154 & 0.167 \\
\hline
\end{tabular}

Notes: SD: standard deviation. 1 . The $p$-value for the differences between $\mathrm{HL}$ types is based on the $\chi^{2}$-test (regarding sex) and analysis of variance (other variables). 2 . Scale ranging from 0 (worst imaginable health) to 10 (best imaginable health). 3. Scale ranging from 1 (least money, least education and worst jobs or no jobs) to 10 (most money, most education and best jobs). 
Table 3. Correlation and regression coefficients predicting number of doctor visits

\begin{tabular}{|c|c|c|c|c|c|c|}
\hline & \multirow[b]{2}{*}{ Zero-order correlation $(p)$} & \multicolumn{3}{|c|}{ Logistic regression (visits versus no visits) } & \multicolumn{2}{|c|}{$\begin{array}{l}\text { Negative binomial model } \\
\text { number of doctor visits }(>0)\end{array}$} \\
\hline & & $B(\mathrm{SE})$ & OR & $p$ & $B(\mathrm{SE})$ & $p$ \\
\hline Intercept & & $1.78(0.43)$ & 5.92 & $<0.001$ & $2.02(0.14)$ & $<0.001$ \\
\hline Age & $0.35\left(<0.001^{\star \star \star}\right)$ & $0.10(0.20)$ & 1.10 & 0.629 & $0.19(0.06)$ & $0.002^{\star *}$ \\
\hline Self-rated health & $-0.25\left(<0.001^{\star \star \star}\right)$ & $-0.21(0.14)$ & 0.82 & 0.137 & $-0.09(0.04)$ & $0.029^{*}$ \\
\hline Sex (female) & $-0.06(0.180)$ & $-0.24(0.23)$ & 0.78 & 0.282 & $-0.02(0.07)$ & 0.754 \\
\hline Self-rated socio-economic status & $-0.19\left(<0.001^{\star \star \star}\right)$ & $-0.33(0.13)$ & 0.72 & $0.009^{\star *}$ & $-0.05(0.04)$ & 0.194 \\
\hline HL Type 1 & $0.02(0.637)$ & $0.38(0.33)$ & 1.47 & 0.238 & $-0.02(0.10)$ & 0.860 \\
\hline HL Type 2 & $-0.06(0.130)$ & $0.06(0.29)$ & 1.06 & 0.834 & $-0.08(0.11)$ & 0.480 \\
\hline HL Type 3 & $0.17\left(<0.001^{\star \star \star}\right)$ & $0.29(0.34)$ & 1.34 & 0.397 & $0.23(0.11)$ & $0.035^{\star}$ \\
\hline HL Type 4 & $-0.10\left(0.012^{\star}\right)$ & Reference & & & Reference & \\
\hline HL Type $1 \times$ Age & & $0.14(0.30)$ & 1.15 & 0.648 & $0.09(0.09)$ & 0.304 \\
\hline HL Type $2 \times$ Age & & $0.04(0.29)$ & 1.05 & 0.880 & $0.07(0.10)$ & 0.491 \\
\hline HL Type $3 \times$ Age & & $0.13(0.31)$ & 1.14 & 0.667 & $0.21(0.11)$ & $0.047^{\star}$ \\
\hline $\mathrm{N}$ & 579 & 579 & & & 468 & \\
\hline
\end{tabular}

Notes: Age, self-rated health and self-rated socio-economic status are $z$-standardised. $B$ (SE): unstandardised regression coefficient (standard error). OR: odds ratio. HL: health literacy. Significance levels: ${ }^{\star} p<0.05,{ }^{\star *} p<0.01,{ }^{\star * \star} p<0.001$. 


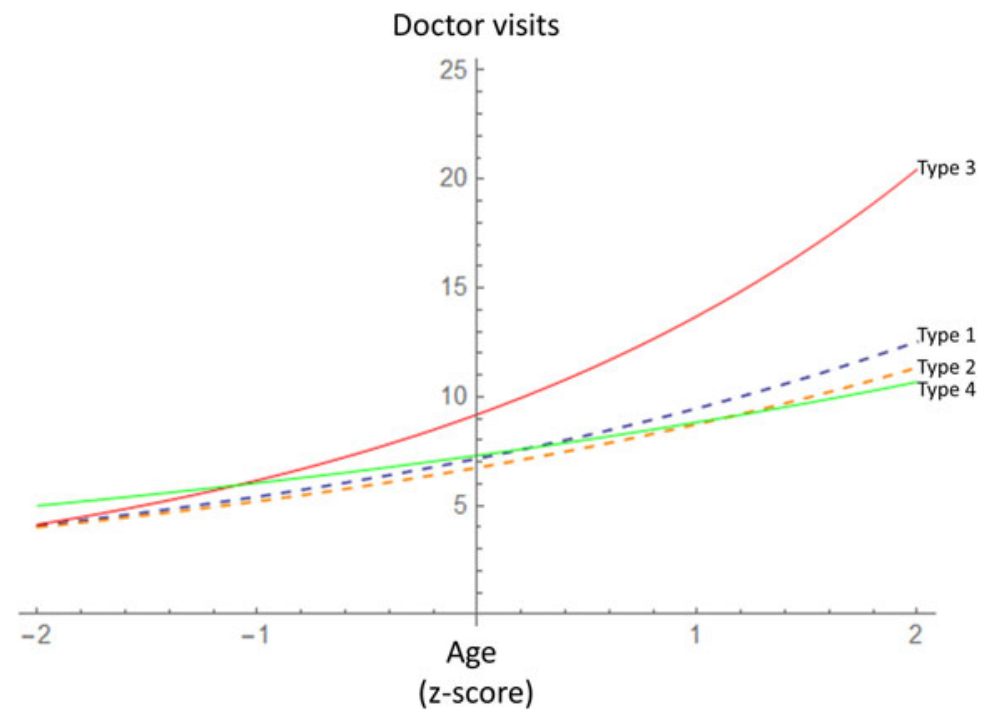

Figure 1. Predicted number of doctor visits per year by age and health literacy type.

To summarise, the results show that individuals classified as HL Type 3 have a significantly higher mean number of doctor visits than HL Type 4 , independently of their age, subjective socio-economic status, subjective health and sex. Moreover, the results show that the number of doctor visits of individuals classified as HL Type 3 increases to a significantly stronger degree with higher age compared to those classified as HL Type 4.

Hence, in accordance with Hypothesis 1, the number of doctor visits increases with age; and, in accordance with $\mathrm{H} 2$, a stronger age-related increase in the number of doctor visits is confirmed for HL Type 3 compared to HL Type 4. Therefore, both hypotheses are confirmed with respect to the number of doctor visits.

Table 4 shows the results of the analyses regarding medication costs.

Similar to the number of doctor visits, bivariate correlations show that medication costs increase with age, worse self-rated health and lower subjective socioeconomic status. In addition, somewhat lower medication costs are observed for females than for males (not significant in analyses weighted by age and sex). With respect to the HL types, only a small significant difference is observed as medication costs of individuals classified as HL Type 1 are slightly higher compared to the others (not significant in analyses weighted by age and sex).

Turning to the multivariate results, the first part of the hurdle model shows that the odds of registered costs for medication in the past year increases as age increases (not significant in analyses weighted by age and sex) and as subjective health worsens.

Similarly, the second part of the model (predicting the amount of medication costs of those with registered costs) shows increasing costs with higher age and worse subjective health. Also, controlling for the other variables, the predicted costs are lower for females than for males. No main (average) effects of HL types 
Table 4. Correlation and regression coefficients predicting medication costs

\begin{tabular}{|c|c|c|c|c|c|c|}
\hline & \multirow[b]{2}{*}{ Zero-order correlation $(p)$} & \multicolumn{3}{|c|}{ Logistic regression (costs versus no costs) } & \multicolumn{2}{|c|}{$\begin{array}{l}\text { Gamma model for medication } \\
\text { costs }(>0)\end{array}$} \\
\hline & & $B(\mathrm{SE})$ & OR & $p$ & $B(\mathrm{SE})$ & $P$ \\
\hline Intercept & & $1.12(0.38)$ & 3.06 & 0.004 & $5.59(0.19)$ & $<0.001$ \\
\hline Age & $0.34\left(<0.001^{\star \star \star}\right)$ & $0.43(0.20)$ & 1.54 & $0.032^{\star}$ & $0.39(0.10)$ & $<0.001^{\star \star \star}$ \\
\hline Self-rated health & $-0.34\left(<0.001^{\star \star \star}\right)$ & $-0.38(0.12)$ & 0.69 & $0.002^{\star *}$ & $-0.34(0.06)$ & $<0.001^{\star \star \star}$ \\
\hline Sex (female) & $-0.13\left(0.002^{\star \star}\right)$ & $0.01(0.20)$ & 1.01 & 0.977 & $-0.32(0.10)$ & $0.002^{\star \star}$ \\
\hline Self-rated socio-economic status & $-0.11\left(0.007^{\star \star}\right)$ & $-0.13(0.11)$ & 0.88 & 0.254 & $0.06(0.05)$ & 0.287 \\
\hline HL Type 1 & $0.08\left(0.045^{\star}\right)$ & $-0.21(0.28)$ & 0.81 & 0.445 & $0.25(0.15)$ & 0.085 \\
\hline HL Type 2 & $-0.02(0.640)$ & $-0.25(0.27)$ & 0.78 & 0.362 & $0.08(0.14)$ & 0.591 \\
\hline HL Type 3 & $0.02(0.656)$ & $0.15(0.32)$ & 1.16 & 0.641 & $-0.08(0.16)$ & 0.626 \\
\hline HL Type 4 & $-0.08(0.051)$ & Reference & & & Reference & \\
\hline HL Type $1 \times$ Age & & $-0.12(0.27)$ & 0.88 & 0.644 & $0.14(0.14)$ & 0.313 \\
\hline HL Type $2 \times$ Age & & $0.20(0.29)$ & 1.22 & 0.492 & $0.09(0.15)$ & 0.575 \\
\hline HL Type $3 \times$ Age & & $0.02(0.29)$ & 1.02 & 0.953 & $0.29(0.14)$ & $0.037^{\star}$ \\
\hline $\mathrm{N}$ & 581 & 581 & & & 412 & \\
\hline
\end{tabular}

Notes: Age, self-rated health and self-rated socio-economic status are $z$-standardised. $B$ (SE): unstandardised regression coefficient (standard error). OR: odds ratio. HL: health literacy. Significance levels: ${ }^{\star} p<0.05,{ }^{\star *} p<0.01,{ }^{\star * \star} p<0.001$. 
are observed. Again, however, a significant interaction effect between age and HL Type 3 is confirmed, which is plotted in Figure 2.

Whereas there are only small differences in medication costs with respect to HL types in younger people (that is, below the sample mean of 52 years), the costs are more diverse for higher regions of individuals' age (above the sample mean of 52 years). In particular, the slope of the association between age and medication costs is steeper for individuals classified as HL Type 3 than it is for others. It is notable that predicted medication costs for individuals classified as HL Type 3 are somewhat lower than those predicted for HL Type 4 in younger age, but will be higher with increasing age.

Therefore, again, both hypotheses are confirmed with respect to medication costs.

\section{Discussion}

The aim of the presented research was to analyse the association between HL and the common increase of health service utilisation (number of doctor visits and medication costs) in later stages of life, as measured by registered data of health service utilisation. Against the background of some inconsistencies shown in previous research, a two-dimensional approach of HL, including the components of subjective HL and health-related knowledge, was employed (Gerich and Moosbrugger, 2018). It was expected that the common increase in health service utilisation will be smallest for individuals with high subjective HL, accompanied by high health-related knowledge (HL Type 4). On the contrary, it was expected that the common increase would be highest for individuals with high subjective HL, accompanied by low health-related knowledge (HL Type 3).

The results confirm our hypotheses with respect to both considered measures of health service utilisation. First, it is confirmed that the number of doctor visits and medication costs increase with higher age. Second, a significant interaction effect between HL type and age is confirmed for both measures of health service utilisation. As shown in Figures 1 and 2, the common age-related increase in health service utilisation is highest for individuals classified as HL Type 3 and lowest for individuals classified as HL Type 4.

Previous research (Gerich and Moosbrugger, 2018) found that the ideal of highly competent and empowered individuals is most likely identified by HL Type 4 (that is, individuals with a combination of high subjective HL and high health-related knowledge). On the contrary, they found that the high subjective HL of individuals classified as HL Type 3 (that is, individuals with a combination of high subjective HL but low health-related knowledge) is more likely to be grounded in a higher unconditional trust in the health-care system and a higher degree of external health-related control. Moreover, they found higher preferences for paternalistic role expectations of doctor-patient interactions, lower willingness for self-medication and less active health-related coping styles for individuals classified as HL Type 3 than for those classified as HL Type 4. Hence, in line with these previous results, the results of the present paper suggest that individuals classified as HL Type 3-despite their high subjective $\mathrm{HL}$ - rely more strongly on professional expertise when faced with health complaints, resulting in a stronger increase in doctor visits with increasing 


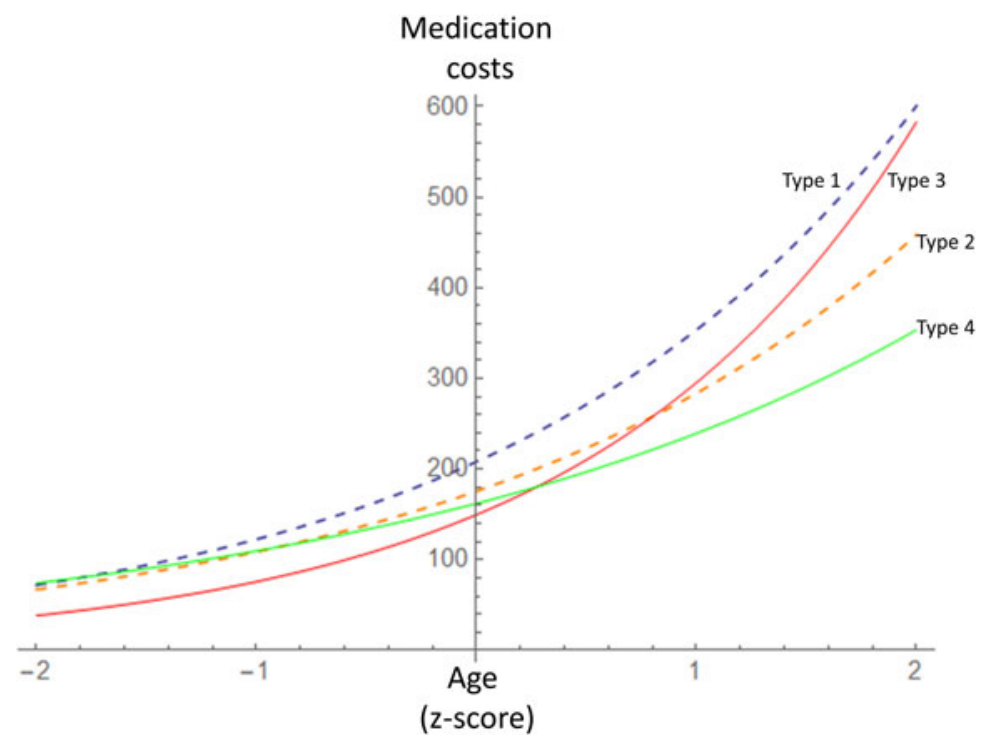

Figure 2. Predicted medication costs ( $€$ per year) by age and health literacy type.

vulnerability in higher ages. Their higher increase in medication costs may also reflect their higher tendency to accept physicians' prescriptions passively. Frisch et al. (2012) point out that high trust in physicians may even prevent individuals with high abilities from active involvement and information seeking. On the other hand, due to their higher trust in medical therapies, it could also be that these types of patients more actively request medications instead of alternative therapies or strategies regarding the adaption of their health-related lifestyles. Similarly, Eichler et al. (2009) concluded from their systematic review that the impact of improved HL on health service utilisation remains unclear, and they suggested two possible scenarios. On one hand, higher HL may lead to reduced health-care utilisation because individuals are enabled to select available options critically and to make health-promoting decisions in their daily lives. On the other hand, improved HL may be associated with greater demands for health-care services, because individuals 'who gain improved skills to retrieve updated health information might behave like uncritical "health care consumers" (Eichler et al., 2009: 321). In the light of our presented results, both scenarios may be true, depending on the definition and measurement of HL. Whereas the first scenario seems to be more likely for health-literate individuals of HL Type 4 in our study, the second scenario may be more likely for health-literate individuals of HL Type 3.

Also, Gerich and Moosbrugger (2018) found that the optimistic view of individuals classified as HL Type 3 regarding the difficulty of health-related tasks is often grounded in a low experience with health complaints and their low concerns about health-related issues. Hence, it is likely that this lower attention and reflection concerning their health state results in a lower frequency of doctor visits in younger ages. On the contrary, with increasing manifest symptoms and chronic diseases associated with higher ages, these individuals are more likely to consult physicians due to their lower engagement and expertise for self-medication. This would 
correspond to our results, which show - especially for the older group (i.e. those above the sample mean age of 52 years) - higher numbers of doctor visits and higher medication costs of individuals classified as HL Type 3 compared to HL Type 4, but a slightly reversed association for the younger age groups. The lower frequencies of health-care utilisation of younger compared to older HL Type 3 individuals than for those classified as HL Type 4 may also reflect possible effects of exacerbation of health conditions in higher ages due to lower attention to health issues in younger ages.

Moreover, although our results show a main (average) effect of HL type on doctor visits, a main conclusion is that the effect of HL depends on age. Therefore, it is recommended that future research not only considers the multi-dimensionality of $\mathrm{HL}$, but also age-specific associations with outcome variables. Our results confirm previous research showing that the well-known age-related increase of health-care utilisation is also accompanied by an increase in variance in higher ages. Because part of this variance is explained by HL, it seems appropriate to concentrate on health promotion measures that focus on HL by addressing health education, empowerment of individuals but also the development of health-literate organisations (Gugglberger, 2019) in order to reduce the financial pressure and to improve the performance of health-care systems in light of the epidemiological transition and ageing of populations.

Although it was not a focus of our research, results showed lower medication costs for women compared to men. This result seems surprising, as past research typically found a higher prevalence for medication prescriptions in females compared to males (Roe et al., 2002; Lehnert et al., 2011). However, in line with our results reversed (Orlando et al., 2020) or no statistical differences (Fernández-Liz et al., 2008) between medication costs and gender were found. Orlando et al. (2020) explained the higher medication costs of males compared to females by the longer duration of treatments and the higher prevalence of diabetes in men compared to women.

Given our results, it can be concluded that single-score measures of HL are not likely to uncover fully the complexity of the mechanisms behind the effects of HL on health-related outcomes and health service utilisation. Therefore, correlational population studies that rely primarily on single-score measures of HL may underestimate the total effects of HL on health-related outcome variables and health service utilisation. Also, single measures of HL that blank out the multi-dimensionality of the construct and relevant boundary conditions such as age may not be suitable to guide interventions effectively. With respect to the patient-physician interaction, for example, additional efforts aimed at empowering patients, fostering their understanding of factors affecting their health or encouraging them to take up a more active role in the management of health may be suitable for individuals with high knowledge and high resourcesfor self-management; this is likely to be the case for patients classified as HL Type 4 in our study. On the other hand, such strategies may disappoint the expectations of other types of patients with a higher demand for medication and professional instructions, as can be expected for patients classified as HL Type 3 in our study. Hence, such interventions may be less effective for such persons, and additional flanking measures such as 'facilitation strategies' (McCormack et al., 2017) would be necessary in order for such groups to be effective. 
Moreover, as limited HL is a function of individual abilities, social contexts (e.g. social support and cohesion) and task difficulty (e.g. barriers embedded in the health-care process), widely claimed interventions regarding the establishment of health-literate organisations, such as the use of easy language for instructions or navigational support, are clearly important to reduce the demands of health-related tasks (Ishikawa and Yano, 2008; Farmanova et al., 2018). However, most of these strategies are primarily designed to address the needs of individuals with both low knowledge and low personal resources, e.g. individuals of HL Type 1 in our study. Therefore, additional strategies to address the various needs of other groups should be taken into account as well. Although our study provides initial insights into the heterogeneity of demands associated with different dimensions of HL, deeper insights are necessary in order to uncover the specific demands of different HL groups in more detail and, thus, guide possible tailored intervention strategies. Future research and development of interventions, for example, could be guided by strategies, as suggested by Beauchamp et al. $(2015,2017)$, who based their design of tailored organisation-based interventions on profiles identified with the help of a cluster analysis that was based on the scores of a multi-dimensional HL inventory. Hypothetical narrative case descriptions (which those authors called 'vignettes') for each identified cluster were used to develop tailored organisation-based interventions in the context of workshops including multi-professional teams.

Our results suggest that individuals categorised as HL Type 3 may have a higher risk of over-medicalisation with increasing age. Jansen et al. (2016) conclude that patients as well as physicians tend to overestimate the benefits of medication and that doctor-patient interactions that follow the ideal of a shared decision model can help to favour the choice of conservative therapeutic options where appropriate. However, as Belcher et al. (2006) confirmed, not all patients have a preference for involvement and therefore they conclude that physicians need to ask patients directly regarding their preferred degree of involvement. Nevertheless, even in cases where patients prefer not to be involved, Belcher et al. (2006) recommend that physicians explore further the reasons for this preference to uncover possible barriers for shared decision interactions. This may, however, conflict with time restrictions of doctor-patient interactions, and physicians may find it 'easier to write the prescription than it is to take a lot of time talking about behavioural modification' (Moloney, 2017: 344). Besides aspects of patient involvement, and especially for patients with low preference for involvement (which can be expected for patients classified as HL Type 3 in our study), physicians play a crucial role in the prevention of over-medication because their recommendations strongly influence patients' decisions between medication and possible alternative strategies (Reeve et al., 2017).

As with all research, a number of limitations have to be considered regarding our presented results. First, our analyses are based on cross-sectional data, which means that questions regarding the causality between $\mathrm{HL}$ and health service utilisation cannot be answered. Especially, it cannot be ruled out that certain patterns of health-care utilisation affect a change in the subjective judgement of HL. Although it would be possible to match our questionnaire data with prospective registered data on health-care utilisation, we do not have respondents' consent to do this. Moreover, although part of our interpretation is based on the assumption that specific types of HL are associated with different trajectories of health-care 
utilisation during the lifecourse, this cannot be examined based on our crosssectional data. Additional longitudinal data would be necessary in order to analyse the individual dynamic of HL and health service utilisation in this respect.

Our study only considered two indicators for health service utilisation: doctor visits and medication costs. MacLeod et al. (2017), for example, pointed out that individuals with low literacy levels tend to bypass less costly services and therefore show higher rates of emergency room visits in reaction to health problems and use fewer preventive services. This would suggest a call for including a broader range of health service utilisation, but also for collecting additional data regarding individuals' rationale behind their health service utilisation. Also, future research may provide deeper insights by differentiating between preventive (such as screenings) and curative health-care utilisation.

Although we elaborated on specific hypotheses with respect to the number of visits of general practitioners, future research may also consider other measures of health-care utilisation such as visits of special physicians and hospital admissions. However, contrary to consultations of a general practitioner, the consultation of special physicians and hospital admission is often based on the referral of general practitioners, and therefore hypotheses regarding the utilisation of these health services are less clear. Individuals with higher HL and higher health-related knowledge, for example, may more often directly consult specialist physicians, whereas consultations with special physicians of those with limited HL or limited health-related knowledge may predominately be grounded in referrals of their general practitioner. Therefore, despite such differences, individuals with different HL types may not differ with respect to the number of visits of special physicians.

Finally, the effects of HL on service utilisation may also be dependent on features of the health-care system and individuals' insurance status (Levine et al., 2018; Goto et al., 2019). The Austrian health-care system is based on a Bismarck system of social insurance and characterised by universal coverage and mostly free provider choice, which may promote the over-utilisation of health-care services (Pichlhöfer and Maier, 2015). Therefore, the effects of HL on health-care service utilisation may be different in Austria than in other countries with other health systems.

\section{Conclusion}

Despite the limitations mentioned above, our research reveals that high subjective HL may be associated with a stronger increase in doctor visits and medication costs in later stages of life. Together with the previously reported results of Gerich and Moosbrugger (2018), our results suggest that in some groups high subjective HL may be grounded in overly relying on medical experts and high trust in the effectiveness of medication, associated with a strong external locus of control and more traditional role expectations concerning doctor-patient interaction, which is not in accordance with the idea of highly empowered patients. Although we believe that the HL typology employed in our study has proven to be a fruitful way of uncovering part of the complexity and heterogeneity of the relationship between HL and health service use, other measurement strategies may still be considered to include the multi-dimensional nature of HL. 
Therefore, it is recommended that future research further should develop and optimise inventories for HL beyond simple single-score measures to account for the complexity of the phenomenon. Moreover, prospective studies are needed to examine further the dynamic of HL and outcome relationships across different stages of the lifecourse.

Acknowledgement. Open access funding provided by Johannes Kepler University Linz. Data collection was kindly supported by the Upper Austrian Sickness Fund.

Financial support. This research received no specific grant from any funding agency, commercial or not-for-profit sectors.

Conflict of interest. The authors declare no conflicts of interest.

Ethical standards. This research was approved by the Upper Austrian Ethics Committee.

\section{References}

Adler NE, Epel ES, Castellazzo G and Ickovics JR (2000) Relationship of subjective and objective social status with psychological and physiological functioning. Preliminary data in healthy white women. Health Psychology: Official Journal of the Division of Health Psychology, American Psychological Association 19, 586-592.

Bähler C, Huber CA, Brüngger B and Reich O (2015) Multimorbidity, health care utilization and costs in an elderly community-dwelling population: a claims data based observational study. BMC Health Services Research 15, Article number: 23 (2015).

Baker DW, Parker RM, Williams MV and Clark WS (1998) Health literacy and the risk of hospital admission. Journal of General Internal Medicine 13, 791-798.

Barber J and Thompson S (2004) Multiple regression of cost data. Use of generalised linear models. Journal of Health Services Research \& Policy 9, 197-204.

Beauchamp A, Buchbinder R, Dodson S, Batterham RW, Elsworth GR, McPhee C, Sparkes L, Hawkins M and Osborne RH (2015) Distribution of health literacy strengths and weaknesses across sociodemographic groups: a cross-sectional survey using the Health Literacy Questionnaire (HLQ). BMC Public Health 15, Article number: 678 (2015).

Beauchamp A, Batterham RW, Dodson S, Astbury B, Elsworth GR, McPhee C, Jacobson J, Buchbinder R and Osborne RH (2017) Systematic development and implementation of interventions to Optimise Health Literacy and Access (Ophelia). BMC Public Health 17, Article number: 230 (2017).

Belcher VN, Fried TR, Agostini JV and Tinetti ME (2006) Views of older adults on patient participation in medication-related decision making. Journal of General Internal Medicine 21, 298-303.

Berens E-M, Vogt D, Ganahl K, Weishaar H, Pelikan J and Schaeffer D (2018) Health literacy and health service use in Germany. Health Literacy Research and Practice 2, e115-e122.

Berkman ND, Davis TC and McCormack L (2010) Health literacy. What is it? Journal of Health Communication 15, 9-19.

Berkman ND, Sheridan SL, Donahue KE, Halpern DJ and Crotty K (2011) Low health literacy and health outcomes: an updated systematic review. Annals of Internal Medicine 155, 97-107.

Bitzer EM (2017) Gesundheitskompetenz messen - Kritischer Blick auf standardisierte Verfahren. Public Health Forum 25, 6-9.

Cho YI, Lee S-YD, Arozullah AM and Crittenden KS (2008) Effects of health literacy on health status and health service utilization amongst the elderly. Social Science \& Medicine 66, 1809-1816.

Diehr P, Yanez D, Ash A, Hornbrook M and Lin DY (1999) Methods for analyzing health care utilization and costs. Annual Review of Public Health 20, 125-144.

Eichler K, Wieser S and Brügger U (2009) The costs of limited health literacy: a systematic review. International Journal of Public Health 54, 313-324.

Farmanova E, Bonneville L and Bouchard L (2018) Organizational health literacy: review of theories, frameworks, guides, and implementation issues. Inquiry: A Journal of Medical Care Organization, Provision and Financing 55, 1-17. 
Fernández-Liz E, Modamio P, Catalán A, Lastra CF, Rodríguez T and Mariño EL (2008) Identifying how age and gender influence prescription drug use in a primary health care environment in Catalonia, Spain. British Journal of Clinical Pharmacology 65, 407-417.

Fleary SA, Joseph P and Pappagianopoulos JE (2018) Adolescent health literacy and health behaviors: a systematic review. Journal of Adolescence 62, 116-127.

Frisch A-L, Camerini L, Diviani N and Schulz PJ (2012) Defining and measuring health literacy: how can we profit from other literacy domains? Health Promotion International 27, 117-126.

Gerich J and Moosbrugger R (2018) Subjective estimation of health literacy. What is measured by the HLS-EU scale and how is it linked to empowerment? Health Communication 33, 254-263.

Goto E, Ishikawa H, Okuhara T and Kiuchi T (2019) Relationship of health literacy with utilization of health-care services in a general Japanese population. Preventive Medicine Reports 14, 100811.

Gugglberger L (2019) The multifaceted relationship between health promotion and health literacy. Health Promotion International 34, 887-891.

Haun JN, Patel NR, French DD, Campbell RR, Bradham DD and Lapcevic WA (2015) Association between health literacy and medical care costs in an integrated healthcare system: a regional population based study. BMC Health Services Research 15, Article number: 249 (2015).

HLS-EU Consortium (2012) Comparative Report of Health Literacy in Eight EU Member States. The European Health Literacy Survey HLS-EU. Available at http://ec.europa.eu/chafea/documents/news/ Comparative_report_on_health_literacy_in_eight_EU_member_states.pdf.

Hoebel J, Müters S, Kuntz B, Lange C and Lampert T (2015) Messung des subjektiven sozialen Status in der Gesundheitsforschung mit einer deutschen Version der MacArthur Scale. Bundesgesundheitsblatt Gesundheitsforschung-Gesundheitsschutz 58, 749-757.

Howard DH, Gazmararian J and Parker RM (2005) The impact of low health literacy on the medical costs of Medicare managed care enrollees. American Journal of Medicine 118, 371-377.

Iacobucci D, Posavac SS, Kardes FR, Schneider MJ and Popovich DL (2015a) The median split: robust, refined, and revived. Journal of Consumer Psychology 25, 690-704.

Iacobucci D, Posavac SS, Kardes FR, Schneider MJ and Popovich DL (2015b) Toward a more nuanced understanding of the statistical properties of a median split. Journal of Consumer Psychology 25, 652-665.

Ishikawa H and Yano E (2008) Patient health literacy and participation in the health-care process. Health Expectations 11, 113-122.

Jansen J, Naganathan V, Carter SM, McLachlan AJ, Nickel B, Irwig L, Bonner C, Doust J, Colvin J, Heaney A, Turner R and McCaffery K (2016) Too much medicine in older people? Deprescribing through shared decision making. BMJ 353, i2893.

Kobayashi LC, Wardle J, Wolf MS and von Wagner C (2016) Aging and functional health literacy: a systematic review and meta-analysis. Journals of Gerontology: Psychological Sciences and Social Sciences 71B, 445-457.

Lehnert T, Heider D, Leicht H, Heinrich S, Corrieri S, Luppa M, Riedel-Heller S and König HH (2011) Review: Health care utilization and costs of elderly persons with multiple chronic conditions. Medical Care Research and Review 68, 387-420.

Levine R, Javalkar K, Nazareth M, Faldowski RA, Ferris MD-G de, Cohen S, Cuttance J, Hooper SR and Rak E (2018) Disparities in health literacy and healthcare utilization among adolescents and young adults with chronic or end-stage kidney disease. Journal of Pediatric Nursing 38, 57-61.

MacLeod S, Musich S, Gulyas S, Cheng Y, Tkatch R, Cempellin D, Bhattarai GR, Hawkins K and Yeh CS (2017) The impact of inadequate health literacy on patient satisfaction, healthcare utilization, and expenditures among older adults. Geriatric Nursing 38, 334-341.

McCormack L, Thomas V, Lewis MA and Rudd R (2017) Improving low health literacy and patient engagement: a social ecological approach. Patient Education and Counseling 100, 8-13.

Mihaylova B, Briggs A, O'Hagan A and Thompson SG (2011) Review of statistical methods for analysing healthcare resources and costs. Health Economics 20, 897-916.

Moloney ME (2017) 'Sometimes, it's easier to write the prescription': physician and patient accounts of the reluctant medicalisation of sleeplessness. Sociology of Health \& Illness 39, 333-348.

Nie JX, Wang L, Tracy CS, Moineddin R and Upshur REG (2010) A population-based cohort study of ambulatory care service utilization among older adults. Journal of Evaluation in Clinical Practice 16, $825-831$. 
Nübling M, Stößel U, Hasselhorn HM, Michaelis M and Hofmann F (2005) Methoden zur Erfassung psychischer Belastungen. Erprobung eines Messinstrumentes (COPSOQ). Dortmund, Germany: NW-Verlag.

Nutbeam D (2008) The evolving concept of health literacy. Social Science \& Medicine 67, 2072-2078.

Orlando V, Mucherino S, Guarino I, Guerriero F, Trama U and Menditto E (2020) Gender differences in medication use: a drug utilization study based on real world data. International Journal of Environmental Research and Public Health 17, 11.

Paasche-Orlow MK and Wolf MS (2007) The causal pathways linking health literacy to health outcomes. American Journal of Health Behavior 31, 19-26.

Palumbo R (2017) Examining the impacts of health literacy on healthcare costs. An evidence synthesis. Health Service Management 30, 197-212.

Parker RM, Ratzan SC and Lurie N (2003) Health literacy: a policy challenge for advancing high-quality health care. Health Affairs 22, 147-153.

Pichlhöfer O and Maier M (2015) Unregulated access to health-care services is associated with overutilization - lessons from Austria. European Journal of Public Health 25, 401-403.

Reeve E, Thompson W and Farrell B (2017) Deprescribing: a narrative review of the evidence and practical recommendations for recognizing opportunities and taking action. European Journal of Internal Medicine 38, 3-11.

Reisi M, Javadzade SH, Heydarabadi AB, Mostafavi F, Tavassoli E and Gholamreza S (2014) The relationship between functional health literacy and health promoting behaviors among older adults. Journal of Education and Health Promotion 3, 119.

Roe CM, McNamara AM and Motheral BR (2002) Gender- and age-related prescription drug use patterns. Annals of Pharmacotherapy 36, 30-39.

Samerski S (2019) Health literacy as a social practice: social and empirical dimensions of knowledge on health and healthcare. Social Science \& Medicine 226, 1-8.

Sørensen K, Van den Broucke S, Fullam J, Doyle G, Pelikan J, Slonska Z and Brand H (2012) Health literacy and public health: a systematic review and integration of definitions and models. BMC Public Health 12, 80 .

Sørensen K, Van den Broucke S, Pelikan JM, Fullam J, Doyle G, Slonska Z, Kondilis B, Stoffels V, Osborne RH and Brand H (2013) Measuring health literacy in populations: illuminating the design and development process of the European Health Literacy Survey Questionnaire (HLS-EU-Q). BMC Public Health 13, 948.

Sørensen K, Pelikan JM, Röthlin F, Ganahl K, Slonska Z, Doyle G, Fullam J, Kondilis B, Agrafiotis D, Uiters E, Falcon M, Mensing M, Tchamov K, van den Broucke S and Brand H (2015) Health literacy in Europe: comparative results of the European health literacy survey (HLS-EU). European Journal of Public Health 25, 1053-1058.

Steckelberg A, Meyer G and Mühlhauser I (2017) Correspondence (letter to the editor): Questionnaire should not be used any longer. Deutsches Ärzteblatt International 114, 330.

Taib NA, Yip C-H and Low W-Y (2011) Recognising symptoms of breast cancer as a reason for delayed presentation in Asian women - the psycho-sociocultural model for breast symptom appraisal: opportunities for intervention. Asian Pacific Journal of Cancer Prevention 12, 1601-1608.

Tiller D, Herzog B, Kluttig A and Haerting J (2015) Health literacy in an urban elderly East-German population - results from the population-based CARLA study. BMC Public Health 15, 883.

Vandenbosch J, Van den Broucke S, Vancorenland S, Avalosse H, Verniest R and Callens M (2016) Health literacy and the use of healthcare services in Belgium. Journal of Epidemiology and Community Health 70, 1032-1038.

Vogt D, Schaeffer D and Berens E-M (2019) Health literacy in later phases of life: findings from Germany and other countries. In Okan O, Bauer U, Levin-Zamir D, Pinheiro P and Sørensen K (eds), International Handbook of Health Literacy: Research, Practice and Policy Across the Lifespan. Bristol, UK: Policy Press, pp. 153-166.

Yang S-C, Luo Y-F and Chiang C-H (2017) The associations among individual factors, eHealth literacy, and health-promoting lifestyles among college students. Journal of Medical Internet Research 19, e15.

Cite this article: Gerich J, Moosbrugger R, Heigl C (2022). Health literacy and age-related health-care utilisation: a multi-dimensional approach. Ageing \& Society 42, 1538-1559. https://doi.org/10.1017/ S0144686X20001609 